\title{
The CCFM uPDF evolution uPDFevolv Version 1.0.00
}

\author{
F. Hautmann ${ }^{1,2,3}$, H. Jung ${ }^{4,5, a}$, S. Taheri Monfared ${ }^{6}$ \\ ${ }^{1}$ Department of Physics and Astronomy, University of Sussex, Brighton BN1 9QH, UK \\ ${ }^{2}$ Rutherford Appleton Laboratory, Chilton OX11 0QX, UK \\ ${ }^{3}$ Department of Theoretical Physics, University of Oxford, Oxford OX1 3NP, UK \\ ${ }^{4}$ DESY, Hamburg, Germany \\ ${ }^{5}$ University of Antwerp, Antwerp, Belgium \\ ${ }^{6}$ School of Particles and Accelerators, Institute for Research in Fundamental Sciences (IPM), P.O.Box 19395-5531, Tehran, Iran
}

Received: 23 July 2014 / Accepted: 15 September 2014 / Published online: 1 October 2014

(C) The Author(s) 2014. This article is published with open access at Springerlink.com

\begin{abstract}
PDFevolvis an evolution code for TMD parton densities using the CCFM evolution equation. A description of the underlying theoretical model and technical realisation is given together with a detailed program description, with emphasis on parameters the user may want to change.
\end{abstract}

\section{Theoretical input}

1.1 CCFM evolution equation and transverse momentum dependent PDFs

QCD calculations of multiple-scale processes and complex final-states require in general transverse-momentum dependent (TMD), or unintegrated, parton density and parton decay functions [1-10]. TMD factorization has been proven recently [1] for inclusive and semi-inclusive deep-inelastic scattering (DIS). Forspecial processes in hadron-hadron scat-

PROGRAM SUMMARY Title of Program: uPDFevolv 1.0.00. Computer for which the program is designed and others on which it is operable: any with standard Fortran 77 (gfortran) and C++, tested on Linux, MAC. Programming Language used: FORTRAN 77, C++. High-speed storage required: No. Separate documentation available: No. Keywords: QCD, small $x$, high-energy factorization, $k_{t^{-}}$ factorization, CCFM, unintegrated PDF (uPDF), transverse momentum dependent PDF (TMD). Nature of physical problem: At high energies collisions of hadrons are described by parton densities dependent on the longitudinal momentum fraction $x$, the transverse momentum $k_{t}$ and the evolution scale $p$ (transverse momentum dependent (TMD) or unintegrated parton density functions (uPDF)). The evolution of the parton density with the scale $p$ valid at both small and moderate $x$ is given by the CCFM evolution equation. Method of solution: Since the CCFM evolution equation cannot be solved analytically, a Monte Carlo approach is applied, simulating at each step of the evolution the full four-momenta of the initial state partonic cascade. Restrictions on the complexity of the problem: None. Other Program used: RoOT for plotting the result. Download of the program: https:// updfevolv.hepforge.org. Unusual features of the program: None.

a e-mail: hannes.jung@desy.de tering, like heavy flavor or heavy boson (including Higgs) production, TMD factorization holds in the high-energy limit $(\operatorname{small} x)$ [11-13].

In the framework of high-energy factorization $[11,14]$ the deep-inelastic scattering cross section can be written as a convolution in both longitudinal and transverse momenta of the TMD parton density function $\mathcal{A}\left(x, k_{t}, \mu\right)$ with off-shell partonic matrix elements, as follows

$\sigma_{j}\left(x, Q^{2}\right)=\int_{x}^{1} d z \int d^{2} k_{t} \hat{\sigma}_{j}\left(x, Q^{2}, z, k_{t}\right) \mathcal{A}\left(z, k_{t}, p\right)$,

with the DIS cross sections $\sigma_{j}(j=2, L)$ related to the structure functions $F_{2}$ and $F_{L}$ by $\sigma_{j}=4 \pi^{2} F_{j} / Q^{2}$. The hard-scattering kernels $\hat{\sigma}_{j}$ of Eq. (1) are $k_{t}$-dependent and the evolution of the transverse momentum dependent gluon density $\mathcal{A}$ is obtained by combining the resummation of small- $x$ logarithmic contributions [15-17] with medium- $x$ and large$x$ contributions to parton splitting [18-20] according to the CCFM evolution equation [21-23].

The factorization formula (1) allows one to resum logarithmically enhanced $x \rightarrow 0$ contributions to all orders in perturbation theory, both in the hard scattering coefficients and in the parton evolution, taking fully into account the dependence on the factorization scale $p$ and on the factorization scheme $[24,25]$.

The CCFM evolution equation [21-23] is an exclusive equation for final state partons and includes finite- $x$ contributions to parton splitting. It incorporates soft gluon coherence for any value of $x$.

\subsubsection{Gluon distribution}

The evolution equation for the TMD gluon density $\mathcal{A}\left(x, k_{t}, p\right)$, depending on $x, k_{t}$ and the evolution variable $p$, is 


$$
\begin{aligned}
& \mathcal{A}\left(x, k_{t}, p\right)=\mathcal{A}_{0}\left(x, k_{t}, p\right)+\int \frac{d z}{z} \int \frac{d q^{2}}{q^{2}} \Theta(p-z q) \\
& \times \Delta_{S}(p, z q) P\left(z, q, k_{t}\right) \mathcal{A}\left(\frac{x}{z}, k_{t}+(1-z) q, q\right),
\end{aligned}
$$

where $z$ is the longitudinal momentum fraction, $q$ is the angular variable and the $\Theta$ function specifies the ordering condition of the evolution [26].

The first term in the right hand side of Eq. (2) is the contribution of the non-resolvable branchings between the starting scale $q_{0}$ and the evolution scale $p$, and is given by

$\mathcal{A}_{0}\left(x, k_{t}, p\right)=\mathcal{A}_{0}\left(x, k_{t}, q_{0}\right) \Delta_{s}\left(p, q_{0}\right)$,

where $\Delta_{S}$ is the Sudakov form factor, and $\mathcal{A}_{0}\left(x, k_{t}, q_{0}\right)$ is the starting distribution at scale $q_{0}$. The integral term in the right hand side of Eq. (2) gives the $k_{t}$-dependent branchings in terms of the Sudakov form factor $\Delta_{S}$ and unintegrated splitting function $P$.

The Sudakov form factor $\Delta_{S}$ is given by

$\Delta_{s}\left(p, q_{0}\right)=\exp \left(-\int_{q_{0}^{2}}^{p^{2}} \frac{d q^{2}}{q^{2}} \int_{0}^{1-q_{0} / q} d z \frac{\bar{\alpha}_{s}\left(q^{2}(1-z)^{2}\right)}{1-z}\right)$,

with $\bar{\alpha}_{S}=C_{A} \alpha_{S} / \pi=3 \alpha_{s} / \pi$.

For application in Monte Carlo event generators, like CASCADE [27,28], it is of advantage to write the CCFM evolution equation in differential form:

$$
\begin{aligned}
& p^{2} \frac{d}{d p^{2}} \frac{x \mathcal{A}\left(x, k_{t}, p\right)}{\Delta_{s}\left(p, q_{0}\right)} \\
& \quad=\int d z \frac{d \phi}{2 \pi} \frac{P\left(z, p / z, k_{t}\right)}{\Delta_{s}\left(p, q_{0}\right)} x^{\prime} \mathcal{A}\left(x^{\prime}, k_{t}^{\prime}, p / z\right),
\end{aligned}
$$

where the splitting variable $x^{\prime}$ is given by $x^{\prime}=x / z, k_{t}^{\prime}=$ $q_{t}(1-z) / z+k_{t}$, and $\phi$ is the azimuthal angle of $q_{t}$.

For the evolution of the parton densities, however, a forward evolution approach, starting from the low scale $q_{0}$ towards the hard scale $p$, is used.

The splitting function $P_{g g}\left(z_{i}, q_{i}, k_{t i}\right)$ for branching $i$ is given by [29] (set by Ipgg $=1, n s=1$ in uPDFevolv)

$$
\begin{gathered}
P_{g g}\left(z_{i}, q_{i}, k_{t i}\right)=\bar{\alpha}_{s}\left(q_{i}^{2}\left(1-z_{i}\right)^{2}\right)\left(\frac{1}{1-z_{i}}-1+\frac{z_{i}\left(1-z_{i}\right)}{2}\right) \\
+\bar{\alpha}_{s}\left(k_{t i}^{2}\right)\left(\frac{1}{z_{i}}-1+\frac{z_{i}\left(1-z_{i}\right)}{2}\right) \Delta_{n s}\left(z_{i}, q_{i}^{2}, k_{t i}^{2}\right)
\end{gathered}
$$

where $\Delta_{n s}$ is the non-Sudakov form factor defined by

$$
\begin{aligned}
\log \Delta_{n s}= & -\bar{\alpha}_{s}\left(k_{t i}^{2}\right) \int_{0}^{1} d z^{\prime}\left(\frac{1}{z^{\prime}}-1+\frac{z^{\prime}\left(1-z^{\prime}\right)}{2}\right) \\
& \times \int \frac{d q^{2}}{q^{2}} \Theta\left(k_{t i}-q\right) \Theta\left(q-z^{\prime} q_{t i}\right)
\end{aligned}
$$

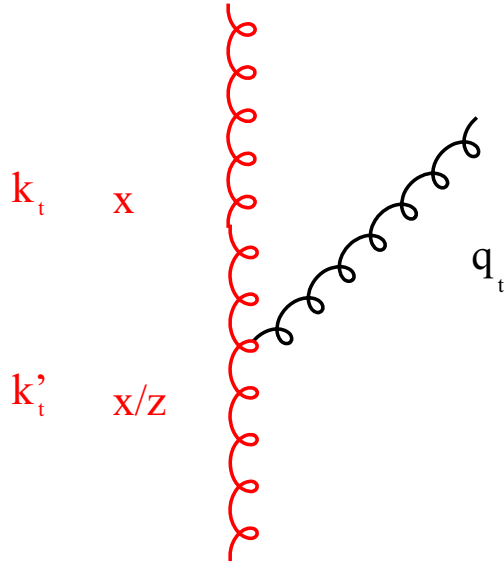

Fig. 1 Gluon branching

In addition to the full splitting function, simplified versions are useful in applications and are made available. One uses only the singular parts of the splitting function (set by Ipgg $=0, \mathrm{~ns}=0$ in uPDFevolv):

$P_{g g}\left(z, q, k_{t}\right)=\frac{\bar{\alpha}_{s}\left(q^{2}\right)}{1-z}+\frac{\bar{\alpha}_{s}\left(k_{t}^{2}\right)}{z} \Delta_{n s}\left(z, q^{2}, k_{t}\right)$

with

$\log \Delta_{n s}=-\bar{\alpha}_{s}\left(k_{t i}^{2}\right) \int_{0}^{1} \frac{d z^{\prime}}{z^{\prime}} \int \frac{d q^{2}}{q^{2}} \Theta\left(k_{t i}-q\right) \Theta\left(q-z^{\prime} q_{t i}\right)$.

Another uses $\alpha_{s}\left(q^{2}\right)$ also for the small $z$ part (set by Ipgg $=2$, $\mathrm{ns}=2$ in uPDFevolv):

$P_{g g}\left(z, q, k_{t}\right)=\frac{\bar{\alpha}_{s}\left(q^{2}\right)}{1-z}+\frac{\bar{\alpha}_{s}\left(q^{2}\right)}{z} \Delta_{n s}\left(z, q^{2}, k_{t}\right)$

with

$\log \Delta_{n s}=-\int_{0}^{1} \frac{d z^{\prime}}{z^{\prime}} \int \frac{d q^{2}}{q^{2}} \bar{\alpha}_{s}\left(q^{2}\right) \Theta\left(k_{t i}-q\right) \Theta\left(q-z^{\prime} q_{t i}\right)$.

In general a four-momentum a can be written in lightcone variables as $\mathbf{a}=\left(a^{+}, a^{-}, a_{T}\right)$ with $a^{+}$and $a^{-}$being the light-cone components and $a_{T}$ being the transverse component. The CCFM (as well as the BFKL) evolution depends only on one of the light-cone components. Assuming that the other one can be neglected, this leads to the condition that the virtuality of the parton propagator $a^{2}=2 a^{+} a^{-}-a_{T}^{2}$ should be dominated by the transverse component, while the contribution from the longitudinal components is required to be small. The condition that $a^{+} a^{-}=0$ leads to the socalled consistency constraint (see Fig. 1), which has been implemented in different forms (set by Ikincut $=1,2,3$ 
in uPDFevolv)

$$
\begin{aligned}
q_{t}^{2} & <\frac{k_{t}^{2}}{z} \operatorname{LDC}[21,30] \\
q_{t}^{2} & <\frac{(1-z) k_{t}^{2}}{z}[31] \\
k_{t}^{\prime 2} & <\frac{k_{t}^{2}}{z} \text { BFKL [31] }
\end{aligned}
$$

\subsubsection{Valence quarks}

Using the method of $[32,33]$ valence quarks are included in the branching evolution at the transverse-momentum dependent level according to

$$
\begin{gathered}
x Q_{v}\left(x, k_{t}, p\right)=x Q_{v 0}\left(x, k_{t}, p\right)+\int \frac{d z}{z} \int \frac{d q^{2}}{q^{2}} \Theta(p-z q) \\
\times \Delta_{s}(p, z q) P_{q q}\left(z, q, k_{t}\right) x Q_{v}\left(\frac{x}{z}, k_{t}+(1-z) q, q\right),(15)
\end{gathered}
$$

where $p$ is the evolution scale. The quark splitting function $P_{q q}$ is given by

$P_{q q}\left(z, q, k_{t}\right)=\frac{C_{F}}{2 \pi} \alpha_{s}\left(q^{2}(1-z)^{2}\right) \frac{1+z^{2}}{1-z}$.

In Eqs. (15), (16) the non-Sudakov form factor is not included, unlike the CCFM kernel given in the appendix B of [22], because we only associate this factor with $1 / z$ terms. The term $x Q_{v 0}$ in Eq. (15) is the contribution of the nonresolvable branchings between starting scale $q_{0}$ and evolution scale $p$, given by

$x Q_{v 0}\left(x, k_{t}, p\right)=x Q_{v 0}\left(x, k_{t}, q_{0}\right) \Delta_{s}\left(p, q_{0}\right)$,

where $\Delta_{S}$ is the Sudakov form factor.

\subsubsection{Sea quarks}

For a complete description of the final states also the contribution from sea-quarks needs to be included. We include splitting functions $P_{a b}$ according to

$$
\begin{aligned}
P_{g g}(z)= & \bar{\alpha}_{s}\left(\frac{1}{1-z_{i}}-1+\frac{z_{i}\left(1-z_{i}\right)}{2}\right) \\
& +\bar{\alpha}_{s}\left(\frac{1}{z_{i}}-1+\frac{z_{i}\left(1-z_{i}\right)}{2}\right) \Delta_{n s} \\
P_{q g}(z)= & \bar{\alpha}_{s} \frac{1}{4 C_{A}}\left(z^{2}+(1-z)^{2}\right) \\
P_{g q}(z)= & \bar{\alpha}_{s} \frac{C_{F}}{2 C_{A}}\left(\frac{1+(1-z)^{2}}{z}\right) \\
P_{q q}(z)= & \bar{\alpha}_{s} \frac{C_{F}}{2 C_{A}}\left(\frac{1+z^{2}}{1-z}\right) \\
\text { with } \bar{\alpha}_{s}= & C_{A} \alpha_{s} / \pi, C_{A}=3 \text { and } C_{F}=4 / 3 .
\end{aligned}
$$

The $g \rightarrow q \bar{q}$ splitting has been calculated in a $k_{t}$ - factorized form in [24],

$$
\begin{aligned}
P_{q g}\left(z, \tilde{q}, k_{t}\right)= & \bar{\alpha}_{s} \frac{1}{4 C_{A}}\left[\frac{\tilde{q}^{2}}{\tilde{q}^{2}+z(1-z) k_{t}^{2}}\right]^{2} \\
& \times\left(z^{2}+(1-z)^{2}+4 z^{2}(1-z)^{2} \frac{k_{t}^{2}}{\tilde{q}^{2}}\right)
\end{aligned}
$$

with $\tilde{q}=q-z k_{t}$, and $q\left(k_{t}\right)$ being the transverse momentum of the quark (gluon).

The evolution equation for the TMD sea-quark density $\mathcal{S}\left(x, k_{t}, p\right)$, depending on $x, k_{t}$ and the evolution variable $p$ is (we allow a general $k_{t}$ dependence of the splitting functions, as proposed in appendix B of [22], even if it is not included in Eqs. (18)-(21)),

$$
\begin{aligned}
& \mathcal{S}\left(x, k_{t}, p\right)=\mathcal{S}_{0}\left(x, k_{t}, p\right) \\
& +\int \frac{d z}{z} \int \frac{d q^{2}}{q^{2}} \Theta(p-z q) \Delta_{s}(p, z q) P_{q g}\left(z, q, k_{t}\right) \\
& \quad \times \mathcal{A}\left(\frac{x}{z}, k_{t}+(1-z) q, q\right) \\
& \quad+\int \frac{d z}{z} \int \frac{d q^{2}}{q^{2}} \Theta(p-z q) \Delta_{s}(p, z q) P_{q q}\left(z, q, k_{t}\right) \\
& \quad \times \mathcal{S}\left(\frac{x}{z}, k_{t}+(1-z) q, q\right),
\end{aligned}
$$

where $\mathcal{S}_{0}\left(x, k_{t}, p\right)$ is the non-resolvable branching probability similar to Eqs. (3), (17).

The evolution of the TMD gluon density including the contribution from quarks is given by

$$
\begin{aligned}
& \mathcal{A}\left(x, k_{t}, p\right)=\mathcal{A}_{0}\left(x, k_{t}, p\right) \\
& +\int \frac{d z}{z} \int \frac{d q^{2}}{q^{2}} \Theta(p-z q) \Delta_{s}(p, z q) P_{g g}\left(z, q, k_{t}\right) \\
& \quad \times \mathcal{A}\left(\frac{x}{z}, k_{t}+(1-z) q, q\right) \\
& +\int \frac{d z}{z} \int \frac{d q^{2}}{q^{2}} \Theta(p-z q) \Delta_{s}(p, z q) P_{g q}\left(z, q, k_{t}\right) \\
& \quad \times \mathcal{S}\left(\frac{x}{z}, k_{t}+(1-z) q, q\right) .
\end{aligned}
$$

\subsubsection{Monte Carlo solution of the CCFM evolution equations}

The evolution equations (23), (24) are integral equations of the Fredholm type

$$
f(x)=f_{0}(x)+\lambda \int_{a}^{b} K(x, y) f(y) d y
$$


and can be solved by iteration as a Neumann series

$$
\begin{aligned}
f_{1}(x)= & f_{0}(x)+\lambda \int_{a}^{b} K(x, y) f_{0}(y) d y \\
f_{2}(x)= & f_{0}(x)+\lambda \int_{a}^{b} K\left(x, y_{1}\right) f_{0}\left(y_{1}\right) d y_{1} \\
& +\lambda^{2} \int_{a}^{b} \int_{a}^{b} K\left(x, y_{1}\right) K\left(y_{1}, y_{2}\right) f_{0}\left(y_{2}\right) d y_{2} d y_{1} \\
& \ldots
\end{aligned}
$$

using the kernel $K(x, y)$, with the solution

$$
f(x)=\lim _{n \rightarrow \infty} \sum_{i=0}^{n} f_{i}(x) .
$$

Applying this to the evolution equations Eqs. (23), (24), we identify $f_{0}$ with the first term in Eq. (24), where we use for simplicity here and in the following $\Delta_{S}(p)=\Delta_{S}\left(p, q_{0}\right)$ :

$\mathcal{A}_{0}\left(x, k_{t}, p\right)=\mathcal{A}_{0}\left(x, k_{t}\right) \Delta_{s}(p)$.

The first iteration involves one branching:

$$
\begin{aligned}
\mathcal{A}_{1}\left(x, k_{t}, p\right)= & \mathcal{A}_{0}\left(x, k_{t}\right) \Delta_{s}(p) \\
& +\int_{x}^{1} \frac{d z^{\prime}}{z^{\prime}} \int_{q_{0}}^{p} \frac{d q^{\prime 2}}{q^{\prime 2}} \Theta\left(p-z^{\prime} q^{\prime}\right) \\
& \times \frac{\Delta_{s}(p)}{\Delta_{s}\left(z q^{\prime}\right)} \tilde{P}\left(z^{\prime}\right) \mathcal{A}_{0}\left(x / z^{\prime}, k_{t}^{\prime}, q^{\prime}\right) .
\end{aligned}
$$

The second iteration involves two branchings,

$$
\begin{aligned}
& \mathcal{A}_{2}(\left.x, k_{t}, p\right)=\mathcal{A}_{0}\left(x, k_{t}\right) \Delta(p) \\
&+\int_{x}^{1} \frac{d z^{\prime}}{z^{\prime}} \int_{q_{0}}^{p} \frac{d q^{\prime 2}}{q^{\prime 2}} \Theta\left(p-z^{\prime} q^{\prime}\right) \\
& \times \frac{\Delta(p)}{\Delta\left(q^{\prime}\right)} \tilde{P}\left(z^{\prime}\right) \mathcal{A}_{1}\left(x / z^{\prime}, k_{t}^{\prime}, q^{\prime}\right) \\
&= \mathcal{A}_{0}\left(x, k_{t}\right) \Delta(p)+\frac{\alpha_{\mathrm{s}}}{2 \pi} \int_{x}^{1} \frac{d z^{\prime}}{z^{\prime}} \int_{q_{0}}^{p} \frac{d q^{\prime 2}}{q^{\prime 2}} \\
& \times \Theta\left(p-z^{\prime} q^{\prime}\right) \frac{\Delta_{s}(p)}{\Delta_{s}\left(z q^{\prime}\right)} \tilde{P}\left(z^{\prime}\right) \mathcal{A}_{0}\left(x / z^{\prime}, k_{t}^{\prime}, q^{\prime}\right) \\
&+\left(\frac{\alpha_{\mathrm{s}}}{2 \pi}\right)^{2} \int_{x}^{1} \frac{d z^{\prime}}{z^{\prime}} \int_{q_{0}}^{p} \frac{d q^{\prime 2}}{q^{\prime 2}} \Theta\left(p-z^{\prime} q^{\prime}\right) \\
& \times \frac{\Delta_{s}(p)}{\Delta_{s}\left(z^{\prime} q^{\prime}\right)} \tilde{P}\left(z^{\prime}\right) \times \int_{x}^{1} \frac{d z^{\prime \prime}}{z^{\prime \prime}} \int_{q_{0}}^{p} \frac{d q^{\prime \prime}}{q^{\prime \prime}} \\
& \times \Theta\left(p-z^{\prime \prime} q^{\prime \prime}\right) \frac{\Delta_{s}(p)}{\Delta_{s}\left(z^{\prime \prime} q^{\prime \prime}\right)} \tilde{P}\left(z^{\prime \prime}\right) \mathcal{A}_{0}\left(z^{\prime \prime} / z^{\prime}, k_{t}^{\prime \prime}, q^{\prime \prime}\right), \\
& \mathcal{A}_{3}\left(x, k_{t}, p\right)=\cdots
\end{aligned}
$$

In a Monte Carlo (MC) solution [34,35] we evolve from $q_{0}$ to a value $q^{\prime}$ obtained from the Sudakov factor $\Delta_{s}\left(q^{\prime}, q_{0}\right)$ (for a schematic visualisation of the evolution see Fig. 2).
Note that the Sudakov factor $\Delta_{S}\left(q^{\prime}, q_{0}\right)$ gives the probability for evolving from $q_{0}$ to $q^{\prime}$ without resolvable branching. The value $q^{\prime}$ is obtained from solving for $q^{\prime}$ :

$R=\Delta_{s}\left(q^{\prime}, q_{0}\right)$,

for a random number $R$ in $[0,1]$.

If $q^{\prime}>p$ then the scale $p$ is reached and the evolution is stopped, and we are left with just the first term without any resolvable branching. If $q^{\prime}<p$ then we generate a branching at $q^{\prime}$ according to the splitting function $\tilde{P}\left(z^{\prime}\right)$, as described below, and continue the evolution using the Sudakov factor $\Delta_{S}\left(q^{\prime \prime}, q^{\prime}\right)$. If $q^{\prime \prime}>p$ the evolution is stopped and we are left with just one resolvable branching at $q^{\prime}$. If $q^{\prime \prime}<p$ we continue the evolution as described above. This procedure is repeated until we generate $q>p$. By this procedure we sum all kinematically allowed contributions in the series $\sum f_{i}(x, p)$ and obtain an MC estimate of the parton distribution function.

With the Sudakov factor $\Delta_{S}$ and using

$$
\begin{aligned}
\frac{\partial}{\partial q^{\prime 2}} \Delta_{s}\left(p, z q^{\prime}\right) & =\frac{\partial}{\partial q^{\prime 2}} \frac{\Delta_{s}(p)}{\Delta_{s}\left(z q^{\prime}\right)} \\
& =\frac{\Delta_{s}(p)}{\Delta_{s}\left(z q^{\prime}\right)}\left[\frac{1}{q^{\prime 2}}\right] \int^{z_{\max }} d z \tilde{P}(z),
\end{aligned}
$$

we can write the first iteration of the evolution equation as

$$
\begin{aligned}
\mathcal{A}_{1}\left(x, k_{t}, p\right)= & \mathcal{A}_{0}\left(x, k_{t}, p\right) \\
& +\int_{x}^{1} \frac{d z^{\prime}}{z^{\prime}} \int_{q_{0}}^{p} d \Delta_{s}\left(p, z^{\prime} q^{\prime}\right) \tilde{P}\left(z^{\prime}\right) \\
& \times \mathcal{A}_{0}\left(x / z^{\prime}, k_{t}^{\prime}, q^{\prime}\right)\left[\int^{z_{\max }} d z \tilde{P}(z)\right]^{-1} .
\end{aligned}
$$

The integrals can be solved by a Monte Carlo method [36]: $z$ is generated from

$$
\int_{z_{\min }}^{z} d z^{\prime} \tilde{P}\left(z^{\prime}\right)=R_{1} \int_{z_{\min }}^{z_{\max }} d z^{\prime} \tilde{P}\left(z^{\prime}\right),
$$

with $R_{1}$ being a random number in $[0,1]$, and $q^{\prime}$ is generated from

$$
\begin{aligned}
R_{2} & =\int_{-\infty}^{x} f\left(x^{\prime}\right) d x^{\prime}=F(x) \\
& =\int_{z q}^{p} \frac{\partial}{\partial q^{\prime 2}}\left(\frac{\Delta_{s}(p)}{\Delta_{s}\left(z q^{\prime}\right)}\right) d q^{\prime 2} \\
& =\Delta_{s}\left(p, z q^{\prime}\right)
\end{aligned}
$$

solving for $q^{\prime}$, using $z$ from above and another random number $R_{2}$ in $[0,1]$.

This completes the calculation on the first splitting. This procedure is repeated until $q^{\prime}>p$ and the evolution is stopped. 

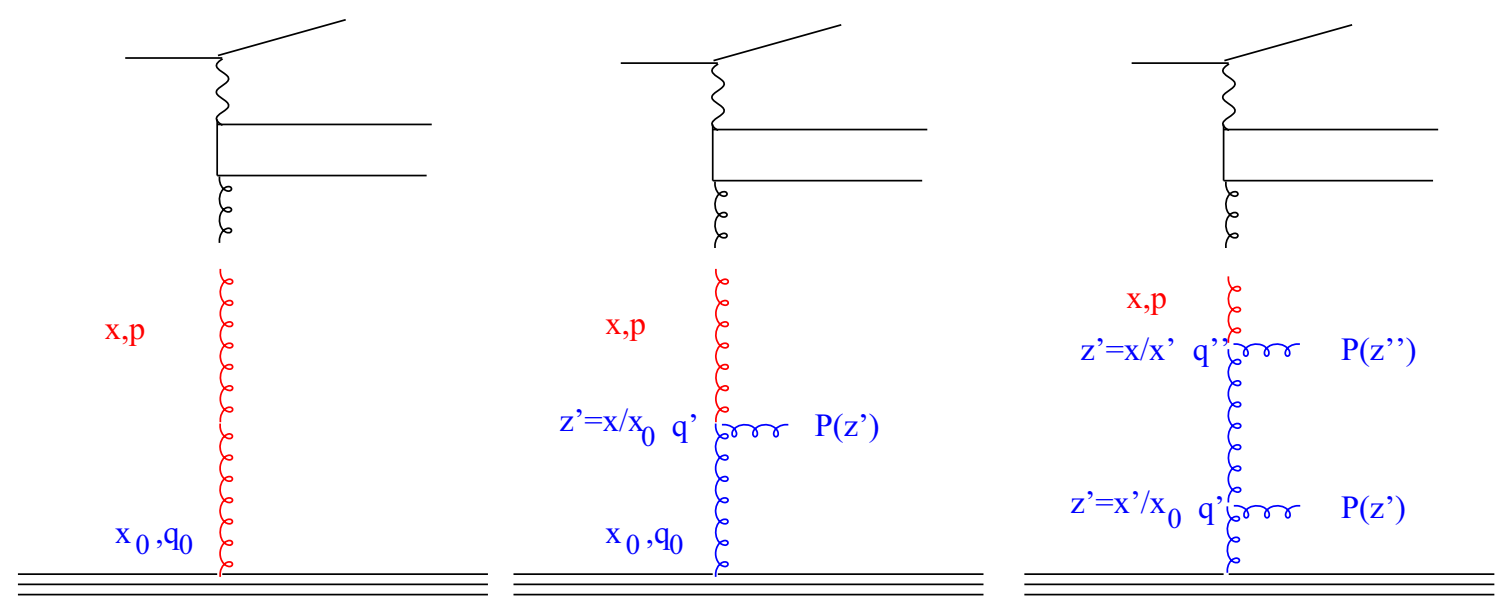

Fig. 2 Evolution by iteration

With $z^{\prime}$ and $q^{\prime}$ selected according to the above the first iteration of the evolution equation yields

$$
\begin{aligned}
& x \mathcal{A}_{1}\left(x, k_{t}, p\right)=x \mathcal{A}_{0}\left(x, k_{t}\right) \Delta_{s}(p) \\
& \quad+\sum_{i} \tilde{P}\left(z_{i}^{\prime}\right) x_{i}^{\prime} \mathcal{A}_{0}\left(x_{i}^{\prime}, k_{t 1}^{\prime}, q_{i}^{\prime}\right)\left[\int^{z_{\max }} d z \tilde{P}(z)\right]^{-1}
\end{aligned}
$$

with $x_{i}^{\prime}=x / z_{i}$.

\subsubsection{Normalisation of gluon and quark distributions}

The valence quark densities are normalised so that they fulfil for every $p$ the flavor sum rule.

The gluon and sea quark densities are normalised so that for every $p$

$$
\begin{aligned}
\int_{0}^{1} d x \int_{0}^{\infty} d k_{t}^{2} x \mathcal{A}\left(x, k_{t}, q_{0}\right)= & \int_{0}^{1} d x \int_{0}^{\infty} d k_{t}^{2}\left(x \mathcal{A}\left(x, k_{t}, p\right)\right. \\
& \left.+x \mathcal{S}\left(x, k_{t}, p\right)\right) .
\end{aligned}
$$

\subsection{Computational techniques: CCFM grid}

When using the CCFM evolution in a fit program to determine the starting distribution $\mathcal{A}_{0}(x)$, a full MC solution $[34,35]$ is no longer suitable, since it is time consuming and suffers from numerical fluctuations. Instead a convolution method introduced in $[37,38]$ is used. The kernel $\tilde{\mathcal{A}}\left(x^{\prime \prime}, k_{t}, p\right)$ is determined once from the Monte Carlo solution of the CCFM evolution equation, and then folded with the non-perturbative starting distribution $\mathcal{A}_{0}(x)$,

$$
\begin{aligned}
& x \mathcal{A}\left(x, k_{t}, p\right)=x \int d x^{\prime} \int d x^{\prime \prime} \mathcal{A}_{0}\left(x^{\prime}\right) \tilde{\mathcal{A}}\left(x^{\prime \prime}, k_{t}, p\right) \delta\left(x^{\prime} x^{\prime \prime}-x\right) \\
& =\int d x^{\prime} \mathcal{A}_{0}\left(x^{\prime}\right) \cdot \frac{x}{x^{\prime}} \tilde{\mathcal{A}}\left(\frac{x}{x^{\prime}}, k_{t}, p\right) .
\end{aligned}
$$

The kernel $\tilde{\mathcal{A}}$ incorporates all of the dynamics of the evolution, including Sudakov form factors and splitting functions. It is determined on a grid of $50 \otimes 50 \otimes 50$ bins in $x, k_{t}, p$. The binning in the grid is logarithmic, except for the longitudinal variable $x$ where we use 40 bins in logarithmic spacing below 0.1 , and 10 bins in linear spacing above 0.1 .

Using this method, the complete coupled evolution of gluon and sea quarks is more complicated, since it is no longer a simple convolution of the kernel with the starting distribution. To simplify the approach, here we allow only for one species of partons at the starting scale, either gluons or seaquarks. During evolution the other species will be generated. This approach, while convenient for QCD fits, has the feature that sea-quarks, in the case of gluons only at $q_{0}$, are generated with perturbative transverse momenta $\left(k_{t}>k_{t}\right.$ cut $)$, without contribution from the soft (non-perturbative) region.

\subsection{Functional forms for starting distribution}

\subsubsection{Standard parametrisation}

For the starting distribution $\mathcal{A}_{0}$, at the starting scale $q_{0}$, the following form is used:

$$
\begin{aligned}
x \mathcal{A}_{0}\left(x, k_{t}, q_{0}\right)= & A_{1} x^{-A_{2}} \cdot(1-x)^{A_{3}}\left(1-A_{4} x\right. \\
& \left.+A_{5} \sqrt{x}+A_{6} x^{2}\right) \exp \left[-k_{t}^{2} / \sigma^{2}\right],
\end{aligned}
$$

with $\sigma^{2}=q_{0}^{2} / 2$ and free parameters $A_{1}, \ldots, A_{6}$.

Valence quarks are treated using the method of $[32,33,38]$ with starting distributions at scale $q_{0}$ parameterized using standard collinear pdfs (set by Ipdf in uPDFevolv) as

$x Q_{v 0}\left(x, k_{t}, q_{0}\right)=x Q_{v \text { coll.pdf }}\left(x, q_{0}\right) \exp \left[-k_{t}^{2} / \sigma^{2}\right]$.

with $\sigma^{2}=q_{0}^{2} / 2$. At every scale $p$ the flavor sum rule is fulfilled for valence quarks. 


\subsubsection{Saturation ansatz}

A saturation ansatz for the starting distribution $\mathcal{A}_{0}$ at scale $q_{0}$ is available, following the parameterisation of the saturation model by Eq. (18) of [39],

$x \mathcal{A}_{s a t}=\frac{1}{\alpha_{s}} \frac{3 \sigma_{0}}{4 \pi^{2}} R_{0}^{2}(x) k_{t}^{2} \exp \left(-R_{0}^{2}(x) k_{t}^{2}\right)$,

with $R_{0}^{2}(x)=\left(x / x_{0}\right)^{\lambda}$. The free parameters are $\sigma_{0}=A_{2}$, $\lambda=A_{3}, x_{0}=A_{4}$ and $\alpha_{s}=A_{5}$. In order to be able to use this type of parameterisation over the full $x$ range, an additional factor of $(1-x)^{A_{6}}$ (see [40]) is applied.

\subsection{Plotting TMDs}

A simple plot program is included in the package. For a graphical web interface use TMDPLOTTER [41].

\subsection{Application}

The evolution of the TMD gluon density has been used to perform fits to the DIS precision data [42,43], as described in detail in [38].

\section{Description of the program components}

2.1 Program history

$\begin{array}{ll}* & \\ \text { uPDFevolv } & \text { Version } 10000 \\ * & \text { first public release } \\ * & \end{array}$

\subsection{Subroutines and functions}

The source code of uPDFevolv and this manual can be found under: https://updfevolv.hepforge.org/
sminit
to initialise
sminfn
to generate starting distributions in $x$ and $k_{t}$
smbran to simulate perturbative branchings
splittgg
splittgq
splittgg
splittqq
szvalnew
smqtem
updfgrid
asbmy (kt) to generate $g \rightarrow g g$ splitting via $P_{g g}$ to generate $q \rightarrow g q$ splitting via $P_{g q}$ to generate $g \rightarrow q \bar{q}$ splitting via $P_{q g}$ to generate $q \rightarrow q g$ splitting via $P_{q q}$ to calculate $z$ values for $g \rightarrow g g$ splitting to generate $t$ from the corresponding Sudakov factor to build, fill and normalise the updf grid. to calculate $\frac{C_{A}}{\pi} \alpha_{S}\left(k_{t}\right)$

Utility routines: evolvetmd main routine to perform CCFM evolution updfread example program to read and plot the results

gadap 1-dimensional Gauss integration routine

gadap2 2-dimensional Gauss integration routine

divdif linear interpolation routine (CERNLIB)

ranlux Random number generator RANLUX (CERNLIB)

2.3 Parameter in steering files

'updf - grid.dat' name of the grid file

oneLoop $=0 \quad$ to select all loop CCFM or one loop DGLAP type evolution

saturation $=0$ to select standard or saturated initial condition

Ipdf $=60500 \quad$ LHApdf set name for collinear valence quark starting distribution

Itarget $=2212$ hadron target ID $(2212=$ proton $)$

$\operatorname{Iglu}=1$

for gluon only evolution

$\operatorname{Ipgg}=1$

parameter for $P_{g g}$ splitting function

$\mathrm{ns}=1$ parameter for treatment of non-sudakov form factor

ikincut $=2 \quad$ flag for consistency constraint

$\mathrm{Qg}=2.2$ starting value $q_{0}$ for perturbative evolution

QCDlam $=0.20 \quad$ value for $\Lambda_{q c d}$

$\mathrm{A} 1, \ldots, \mathrm{A} 6$ values for starting distribution; meaning depends on whether standard or saturation ansatz is used.

\section{Example program}

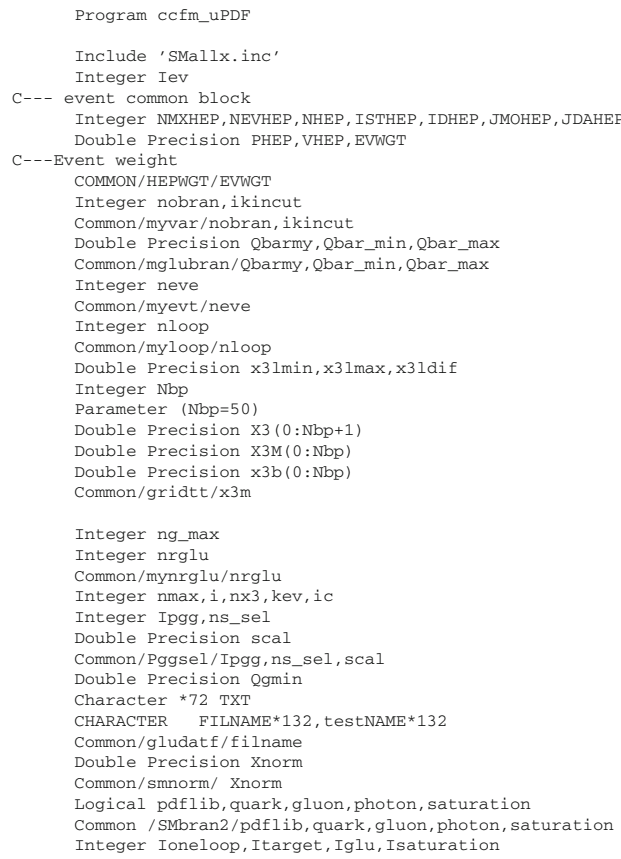


Integer Ipdf

Common/pdf/Ipd

Integer iparton

Common /sMquark/iparton

Character *15 char

Double Precision BB

Double precision ininorm $(-6: 6)$

Common/smininorm/ininorm

Integer IRR

Couble precision au

Logical first

Common/f2fit/au (50), firs

Read $(5, *)$ filname

Write $(6, *)$ ' output file',filname

xnorm $=1$

Read $(5,101)$ TXT

Read(txt, 1005) char, Ioneloop

1005

Write $(6, *)$ txt, char, Ioneloop

Read $(5,101) \mathrm{TX}$

Read (txt, 1010) char, Isaturation

1010 format $(a 14, I 8)$

Write $\left(6,{ }^{*}\right)$ txt, char, Isaturation

Read $(5,101)$ TXT

Read(txt, 1006) char, Ipdf

1006 format (a7, I8)

Read $(5,101)$ TXT

Read (txt, 1007) char, Itarget

Write $(6$ * $)$ txt, char, Itarget

1007 format $(a 10$, I8)

$\operatorname{Read}(5,101)$ TXT

Read(txt, 1008) char, Iglu

Write $(6, *)$ txt, char, Iglu

1008 format $(a 7$, I8)

Read $(5,101)$ TXT

1 Format (A72)

Read(txt, 1000) char, Ipgg

Write $(6, *)$ txt, char, Ipgg

1000

Read $(5,101)$ TXT

Read(txt,1001) char, ns_sel

Write $(6, *)$ txt, char,ns_sel

1001 format $(a 5$, I 8$)$

Read $(5,101)$ TXT
Read (txt, 1011) char, ikincut

Read(txt, 1011) char, ikincut
Write $(6, *)$ txt, char, ikincut

1011 format $(\mathrm{a} 10, \mathrm{I} 8)$

Read $(5,101)$ TXT

Read(txt, 1002) char, Qg

Write $(6, *)$ txt, char, Qg

1002 format (a5, F16.8)

Read $(5,101)$ TXT

Read (txt, 1003) char,

Read $(6, *)$ tat, char, 2

1003 format (a5, F16.8)

Read $(5,101)$ TXT

Rriad $(6, *)$ txt, char, QCDlan

1018 format (a9, F16.8)

$\operatorname{Read}(5,101)$ TXT

Read (txt, 1003)

Read $(5,101)$ TXT

Read $(5,101)$ TXT

Read(txt, 1003)

Read $(5,101)$ TXT

Read (txt, 1003) char, AU (4)

$\operatorname{Read}(5,101)$ TX

Read (txt, 1003) char, AU (5)

$\operatorname{Read}(5,101)$ TX

Read (txt, 1003) char, AU (6)

If(iglu.ne.0) then gluon $=$.true else

gluon $=$. false

If (Ioneloop.eq.1) then

onel $=$. true.

else

onel=. false

If (Isaturation. eq. 1) then

saturation $=$. true.

else

sat

glu.eq. 0) then

Read (txt, 1009) char, Iparton

Write $(6, *)$ txt, char, Iparto

1009 Format (a9, I8)

Endif

Close (50)

C---Initialize run

Call SMinit

neve $=0$ $\operatorname{xini}=0$.

output file, filname

Write $(6, *)$ ' selection Ipgg $=$ ', Ipgg,' ns_sel $=$ ', ns_sel

Write $\left(6,{ }^{*}\right), \quad \mathrm{Qg}={ }^{\prime}, \mathrm{Qg},{ }^{\prime} \mathrm{Qs}={ }^{\prime}, \mathrm{Qs},{ }^{\prime}$ Xnorm $={ }^{\prime}$, Xnorm

Write $(6, *)$, LHAPDFLIB for val quark Ipdf $=1$, Ipdf

Write $(6, *)$, Itarget $=$ ', Itarget
Write $(6, *), \quad \mathrm{BB}=\mathrm{B}_{\mathrm{BB}}$

Qgmin $=\max (\mathrm{Qg}-0.5 \mathrm{~d} 0, \mathrm{QCD} l \mathrm{am}$

Qgmin $=\max (\mathrm{Qg}, \mathrm{QCD}$ lam

$\times 3 \operatorname{lmin}=\log ($ Qgmin $)$

$\times 3 \max =\log (\mathrm{qmax})$

x3ldif $=(x 31 \max -x 31 \mathrm{~min}) /$ Real $(\mathrm{Nbp})$

Do $I=0, \mathrm{Nbp}+1$

$x 3(I)=\exp (x 3 \operatorname{Imin}+x 3 \operatorname{ldif} * \operatorname{Real}(I))$

Enddo

Do $I=0, N b p$

$x 3 m(i)=(x 3(i)+x 3(i+1)) / 2$

Enddo

$\mathrm{N} \times 3=-1$

C---Initialize analysis

Xini $=0$.

Call updfgrid(1)

$\mathrm{N} \times 3=49$

$600 \mathrm{~N} \times 3=\mathrm{N} \times 3+$

write $(6, *), n g$ max $=$, ng $\max$, at $n \times 3-1, n \times 3-1$

IF $(\mathrm{N} \times 3 . g t . \mathrm{Nbp})$ Then

write $\left(6,{ }^{*}\right)$ ' evolve_tmd: Nx3 gt Npb $\rightarrow$ Program stopped' Stop

Qbarmy $=\times 3 m(N \times 3)$

nloop $=\mathrm{N} \times 3$

$n_{\text {n } \max }=0$

Write $\left(6,{ }^{*}\right)$ 'Qbar_my ', Qbarmy

Do Iparton $=0,2$

Write $(6, *)$ evolving parton ID: ', Iparton

If(iparton.eq.0) then

gluon=. true

else

gluon=. false.

Endif

$\mathrm{Xini}=0$

Do $I=1$, nev

C---Initialize

Call SMinfn

C---gluon branching process

Call smbran

If (ng_max.le.nrglu) ng_max=nrglu

NE. 0.0) then

$k e v=i$

if (kev.gt.0) ic $=100000$

if (mod(kev, ic) .eq. 0 ) write $(6, *)$ ' event ', kev, nev,'

Enco

Endif

C---Terminate analysis

call updfgrid(3)

Stop

80 Write $\left(6,{ }^{*}\right)$, steering file ccfm_updf not found,

End

\section{Program installation}

uPDFevolv follows the standard AUTOMAKE convention. To install the program, do the following

1) Get the source

tar xvfz uPDFevolv-XXXX.tar.gz

cd UPDFevolv-XXXX

2) Generate the Makefiles (do not use shared libraries)

./configure

3) Compile the binary

make

4) Install the executable

make instal

4) The executable is in bin

run it with:

bin/updf evolve < steer_gluon-JH-2013-set2

plot the result with:

bin/updfread 
Acknowledgments We are very grateful to Bryan Webber for careful reading of the manuscript and clarifying comments.

Open Access This article is distributed under the terms of the Creative Commons Attribution License which permits any use, distribution, and reproduction in any medium, provided the original author(s) and the source are credited.

Funded by $\mathrm{SCOAP}^{3}$ / License Version CC BY 4.0.

\section{References}

1. J. Collins, Foundations of Perturbative QCD, vol. 32. Cambridge Monographs on Particle Physics, Nuclear Physics and Cosmology (2011)

2. S.M. Aybat, T.C. Rogers, Phys. Rev. D 83, 114042 (2011). arXiv: 1101.5057

3. M. Buffing, P. Mulders, A. Mukherjee, Int. J. Mod. Phys. Conf. Ser. 25, 1460003 (2014). arXiv: 1309.2472

4. M. Buffing, A. Mukherjee, P. Mulders, Phys. Rev. D 88, 054027 (2013). arXiv:1306.5897

5. M. Buffing, A. Mukherjee, P. Mulders, Phys. Rev. D 86, 074030 (2012). arXiv:1207.3221

6. P. Mulders, Pramana 72, 83 (2009). arXiv:0806.1134

7. S. Jadach, M. Skrzypek, Acta Phys. Pol. B 40, 2071 (2009). arXiv:0905.1399

8. F. Hautmann, Acta Phys. Pol. B 40, 2139 (2009)

9. F. Hautmann, M. Hentschinski, H. Jung (2012). arXiv:1205.6358

10. F. Hautmann, H. Jung, Nucl. Phys. Proc. Suppl. 184, 64 (2008). arXiv:0712.0568

11. S. Catani, M. Ciafaloni, F. Hautmann, Nucl. Phys. B 366, 135 (1991)

12. S. Catani, M. Ciafaloni, F. Hautmann, Phys. Lett. B 307, 147 (1993)

13. F. Hautmann, Phys. Lett. B 535, 159 (2002). hep-ph/0203140

14. S. Catani, M. Ciafaloni, F. Hautmann, Phys. Lett. B 242, 97 (1990)

15. L. Lipatov, Phys. Rep. 286, 131 (1997). hep-ph/9610276

16. V.S. Fadin, E. Kuraev, L. Lipatov, Phys. Lett. B 60, 50 (1975)

17. I.I. Balitsky, L.N. Lipatov, Sov. J. Nucl. Phys. 28, 822 (1978)

18. V.N. Gribov, L.N. Lipatov, Sov. J. Nucl. Phys. 15, 438 (1972)

19. G. Altarelli, G. Parisi, Nucl. Phys. B 126, 298 (1977)
20. Y.L. Dokshitzer, Sov. Phys. JETP 46, 641 (1977)

21. M. Ciafaloni, Nucl. Phys. B 296, 49 (1988)

22. S. Catani, F. Fiorani, G. Marchesini, Nucl. Phys. B 336, 18 (1990)

23. G. Marchesini, Nucl. Phys. B 445, 49 (1995). hep-ph/9412327

24. S. Catani, F. Hautmann, Nucl. Phys. B 427, 475 (1994). hep-ph/9405388

25. S. Catani, F. Hautmann, Phys. Lett. B 315, 157 (1993)

26. F. Hautmann, H. Jung, JHEP 10, 113 (2008). arXiv:0805.1049

27. H. Jung, G.P. Salam, Eur. Phys. J. C 19, 351 (2001). hep-ph/0012143

28. H. Jung, S. Baranov, M. Deak, A. Grebenyuk, F. Hautmann et al., Eur. Phys. J. C 70, 1237 (2010). arXiv: 1008.0152

29. M. Hansson, H. Jung (2003). arXiv:hep-ph/0309009

30. B. Andersson, G. Gustafson, J. Samuelsson, Nucl. Phys. B 467, 443 (1996) (revised version)

31. J. Kwiecinski, A.D. Martin, P. Sutton, Z. Phys. C 71, 585 (1996). hep-ph/9602320

32. M. Deak, F. Hautmann, H. Jung, K. Kutak, Forward-Central Jet Correlations at the Large Hadron Collider (2010). arXiv: 1012.6037

33. M. Deak, F. Hautmann, H. Jung, K. Kutak, Eur. Phys. J. C 72, 1982 (2012). arXiv:1112.6354

34. G. Marchesini, B. Webber, Nucl. Phys. B 349, 617 (1991)

35. G. Marchesini, B. Webber, Nucl. Phys. B 386, 215 (1992)

36. F. James, Rep. Prog. Phys. 43, 1145 (1980)

37. H. Jung, F. Hautmann (2012). arXiv: 1206.1796

38. F. Hautmann, H. Jung, Nucl. Phys. B 883, 1 (2014). arXiv: 1312.7875

39. K. Golec-Biernat, M. Wusthoff, Phys. Rev. D 60, 114023 (1999). hep-ph/9903358

40. A. Grinyuk, A. Lipatov, G. Lykasov, N. Zotov, Phys. Rev. D 87, 074017 (2013). arXiv:1301.4545

41. H. Jung et al., TMDlib and TMDplotter. DESY-14-059

42. H1 and ZEUS Collaboration, F. Aaron et al., JHEP 1001, 109 (2010). arXiv:0911.0884

43. H1 and ZEUS Collaboration, H. Abramowicz et al., Eur. Phys. J. C 73, 2311 (2013). arXiv:1211.1182 